\title{
Realizing the E-Wall Concept for Indoor 5G
}

\author{
Marzieh SalarRahimi \\ ESAT-TELEMIC/Smart Antenna Solutions \\ KU Leuven/NXP Semiconductors \\ Leuven, Belgium/ Nijmegen, The Netherland \\ marzieh.salarrahimi@kuleuven.be
}

\author{
Marcel Geurts \\ Smart Antenna Solutions \\ NXP Semiconductors \\ Nijmegen, The Netherland \\ marcel.geurts@nxp.com
}

\author{
Guy A. E. Vandenbosch \\ ESAT-TELEMIC \\ KU Leuven \\ Leuven, Belgium \\ guy.vandenbosch@kuleuven.be
}

\begin{abstract}
The so-called e-wall is a recently introduced concept with the implementation goal of making a flexible and costefficient infrastructure for next generation wireless communication systems in indoor environments. This paper discusses the importance of the e-wall concept and presents some initial attempts to realize it.
\end{abstract}

Keywords-beam steering, antenna integration, mm-wave propagation, active array

\section{INTRODUCTION}

Although moving from traditional microwave frequencies to $\mathrm{mm}$-waves enables the use of more spectrum to support greater data traffic [1], serious propagation challenges arise. Significantly increased propagation, shadowing and penetration losses reduce the coverage dramatically. Many $\mathrm{mm}$-wave channel measurement campaigns have recently been performed to assess channel characteristics in both outdoor and indoor environments $[2,3,4]$. Nevertheless, in most scenarios outdoor cellular infrastructures could not cover indoor environments. Therefore, the undeniable significant role of indoor base transceiver stations (BTSs) has to be considered in 5G communication networks.

In future, one can envisage rooms, even complete houses/offices where the walls are covered with a dense network of antenna elements, realizing indoor BTSs. These antenna arrays can be applied to the walls by implementing them on flexible so-called "e-wall-paper". This type of infrastructure should be simple, targeting cheap mass-produced wall-mounted future indoor telecom applications [5].

To his goal, antenna array design concepts and mm-wave manufacturing technologies have to be investigated to realize the aforementioned features. Embedding of integrated circuits (ICs), interconnects and radiating elements is one of the key research challenges.

In this work, we address the challenges, discuss several possibilities, potentials and limitations. In addition, we present our initial attempts to realize the e-wall concept. A passive beam switching and an active beam forming $2 \times 2$ sub-array are introduced. These simple unit cells can be used as a unit cell to develop a larger array.

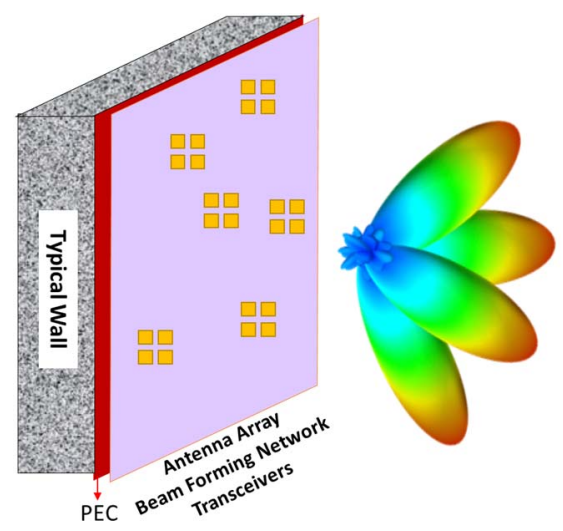

Fig. 1. Realizing e-wall-paper by a sparse antenna array driven by a feeding network and transceivers

\section{THE E-WALL REQUIREMENTS}

There are several requirements that are believed to be crucial for the e-wall paper. First of all, the contained array needs to be beam steerable in the whole hemispheric twodimensional space of possible directions. Beam-forming plays a pivotal role in 5G infrastructures to optimizing the link budget, supporting more users by adding spatial domain diversity, and minimizing interferences. Second, it would be highly beneficiary if this future infrastructure could be deployed in a modular way, using a sub-array as a building block to construct medium sized or larger antenna arrays where appropriate. Third, the array needs to be electromagnetically decoupled from the walls, since the type of wall can be quite diverse. Fourth, since mass deployment is targeted, the sub-array should be as cheap as possible [5].

The e-wall holds an array which contains antenna elements driven by embedded integrated circuits. The antenna element topology, the array configuration and fabrication technology are considered in order to fulfil the aforementioned requirements.

\section{A. Antenna Element Topology and Array Configuration}

In order to electromagnetically decouple the array from the walls, a metal layer can be used. Besides, one another metal layer also forms a reasonable approach to minimize interactions between the radiating parts and the embedded circuits. Accordingly, unidirectional antennas have to be used in the ewall paper. Based on the IC's output type, which can be 


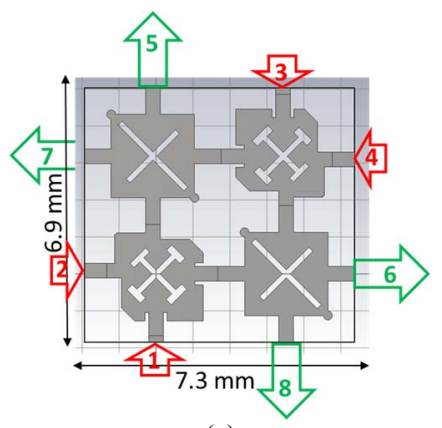

(a)

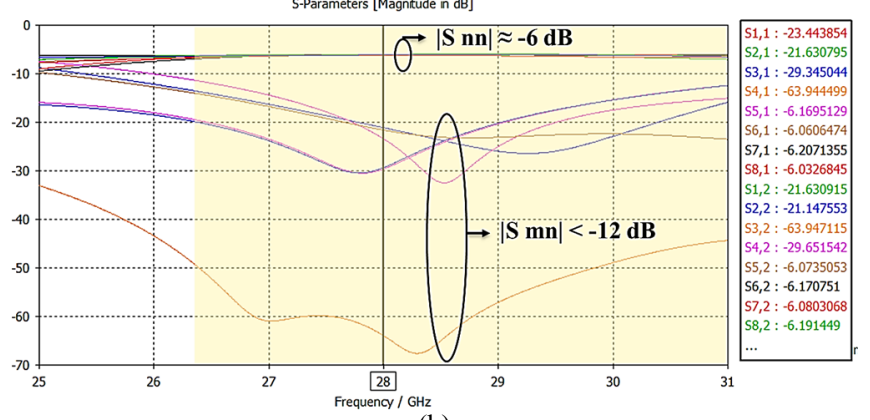

(b)
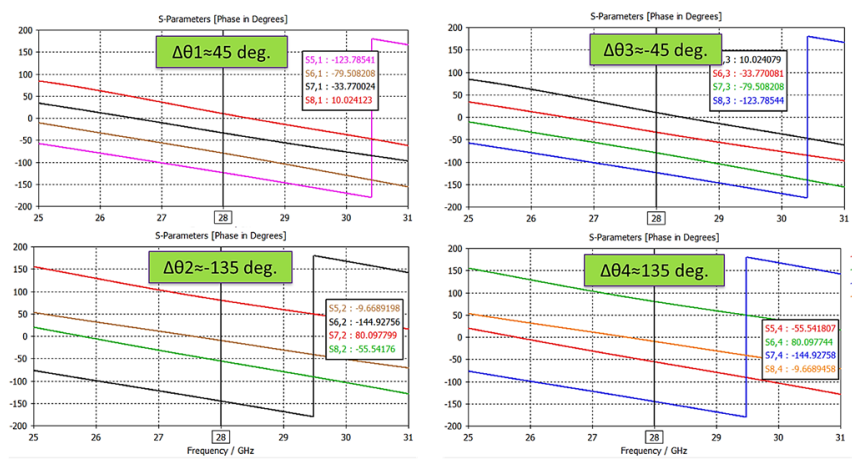

(c)

Fig. 2. The passive beam forming network to feed a beam switching subarray at $28 \mathrm{GHz}$, (a) topology, (b) simulated amplitude of S-parameters, (c) simulated angle of S-parameters, fulfilling $\pm 45^{\circ}$ and $\pm 135^{\circ}$ phase differences.

differential or single ended, modified dipoles or patches are good choices. In the presented examples, we have chosen two kinds of patch antennas.

Another important parameter is configuration of the array. Besides the array's ability to form the desired beams, its ability in heat removal is a challenge that should be addressed by a suitable array configuration. Sparsity is among the most promising approaches to manage the heat removal without using large and bulky heat sinks [6]. Therefore, the e-wall paper holds an array which forms a sparse-array topology, see Fig. 1.

\section{B. Fabrication Technology}

To realize the e-wall paper appropriate fabrication technologies have to be chosen. Cost is a very crucial factor that must be taken into account, besides the minimum power dissipation. Since, the e-wall concept must be realized without using bulky and complicated heat removal systems, the array's power dissipation and interconnection losses must be

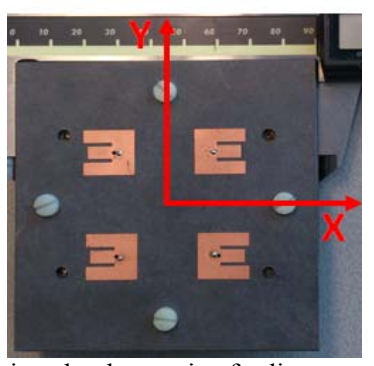

Fig. 3. A sub-array driven by the passive feeding network. The prototype at $6 \mathrm{GHz}$ has been realized by a pretty cost efficient PCB technology.

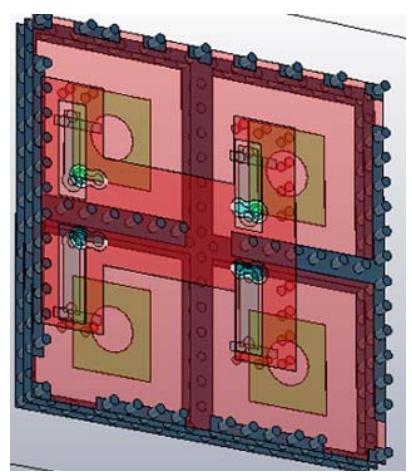

Fig. 4. The Antenna model for the active sub-array, designed satisfying the PCB rules.

minimized. To achieve these goals, not only highly efficient power amplifiers are needed, but also the antennas and ICs should be co-designed.

\section{E-WALL REALIZATION}

In this section, we present our initial attempts to realize the e-wall concept. To fulfil the modularity requirement, we focus on design of the sub-arrays. A passive beam switching and an active beam forming $2 \times 2$ antenna sub-array so far have been designed, realized, and measured.

\section{A. Passive Beam-Switching Sub-Array}

A very simple and passive sub-array is proposed. A passive beam forming network (BFN) is designed at $28 \mathrm{GHz}$ to drive four antenna elements, see Fig. 2. The BFN is a $4 \times 4$ Butler matrix, which equally divides each input signal between four outputs, and with the proper phase differences. Four directional beams can be produced, depending on the selected input port of the BFN. The design has been tuned to be realized by multilayer PCB technology, which is pretty cost efficient. A scaled prototype operating at $6 \mathrm{GHz}$ has been realized and measured in [5], proving the validity of the concept, see Fig. 3.

\section{B. Active Analog Beam-Forming Sub-Array}

An active array module at $28 \mathrm{GHz}$ is presented. The module was designed using a 4-layer PCB stack. As shown in Fig. 4, the antenna structure is implemented on four metal layers, which were connected by three kinds of via; through hole, blind and buried. Regarding the feasible aspect ratio in PCB technology, a minimum via diameter of $250 \mu \mathrm{m}$ with $650 \mu \mathrm{m}$ pitch was kept 


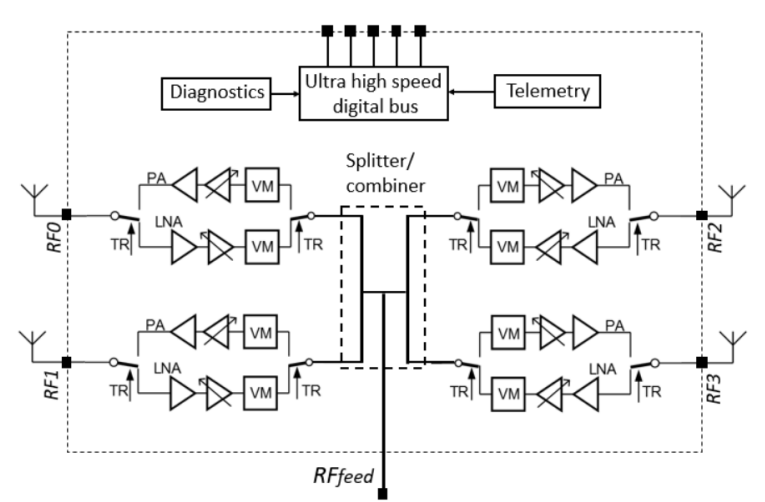

(a)

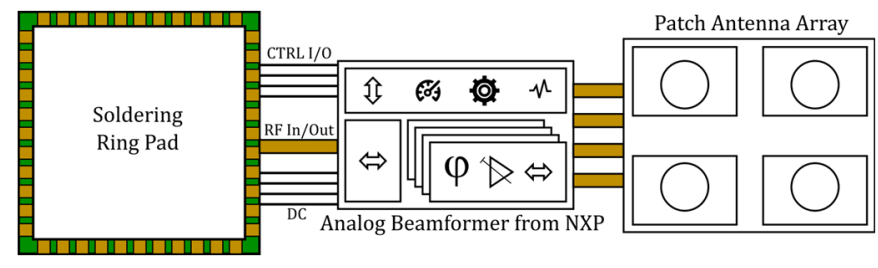

(b)

Fig. 5. Block diagram of the 4-channel beamforming system. (a) Used ABF IC, (b) Antenna module is driven by the ABF

as a design constraint to simplify manufacturing and reduce fabrication costs, consequently.

The board's top layer is connected to an in-house designed 4-channel analog beamforming IC. In addition, there is a soldering ring pad on the top layer that enables connections to the outside world. The second layer is a ground plane which provides isolation between the active components and the radiating elements. The radiating elements are four proximity coupled patches of size $22 \times 24 \mathrm{~mm}^{2}$.

The block diagram of the antenna array architecture is depicted in Fig. 5. To achieve beam steering capabilities, the Analog Beamformer Transceiver from NXP was used. More details about the design are given in [7].

The sub-array which is able to steer the beam in two dimensions was realized and measured successfully. See the fabricated module in Fig. 6 and the measured radiation pattern in Fig. 7. The module was evaluated under a broadside configuration and with its beam steered to +15 and +30 degrees in horizontal direction ( $\mathrm{E}$ plane). The measured radiation patterns in general follow the simulation curves very well. However, the presence of the RF input cable causes a small ripple as the measurement angle reaches -50 degrees.

\section{CONCLUSION}

In this paper, some initial attempts to realize the recently introduced concept of e-wall are presented. We have targeted $\mathrm{mm}$-wave indoor wireless communication systems and the main goal is to realize a high-performance and cost-efficient infrastructure. Therefore, both the passive beam-switching and the active analog beam-forming arrays have been fabricated with conventional PCB technology.

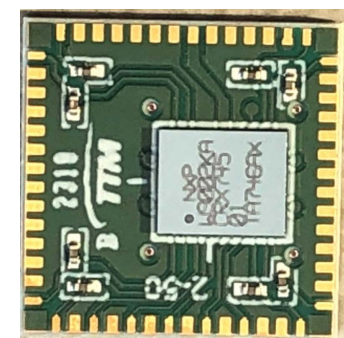

(a)

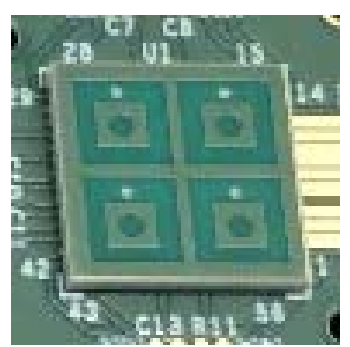

(b)

Fig. 6. Assembled array; (a) top view, (b) bottom view

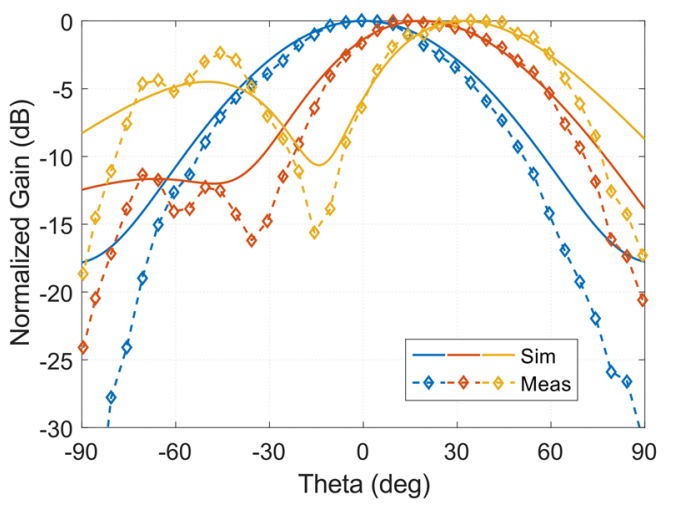

Fig. 7. The antenna's ability to steer the beam in E-plane

\section{ACKNOWLEDGMENT}

This project has received funding from the European Union's Horizon 2020 research and innovation programme under the Marie Sklodowska-Curie grant agreement No. 721732 .

\section{REFERENCES}

[1] T. S. Rappaport et al., "Millimeter wave mobile communications for $5 \mathrm{G}$ cellular: It will work!" IEEE Access, vol. 1, pp. 335-349, May 2013.

[2] S. Hur, S. Baek, B. Kim, Y. Chang, A.F. Molisch, T.S. Rappaport, K. Haneda, and J. Park, "Proposal on millimeter-wave channel modeling for 5G cellular system," IEEE Journal of Selected Topics in Signal Processing, vol. 10, no. 3, pp.454-469, 2016.

[3] K. Haneda et al., "Radio channel sounding campaigns in EU H2020 mmMAGIC project for 5G channel modeling," in Conference Proceedings of The 2016 International Symposium on Antennas and Propagation, The Institute of Electronics, Information and Communication Engineers (IE-ICE), pp. 596-597, Oct. 2016.

[4] A. Lahuerta-Lavieja, M. Johansson, G.A.E. Vandenbosch, and U. Gustavsson, "Environment-embedded radiation patterns at millimeterwave frequencies," in 13th European Conference on Antennas and Propagation (EuCAP 2019), Krakow, Poland, Mar. 2019.

[5] M. SalarRahimi, and G.A.E Vandenbosch, " Beam steerable subarray with small footprint for use as building block in wall-mounted indoor wireless infrastructure," IET Microwaves, Antennas \& Propagation, Jan. 2019.

[6] Y. Aslan, J. Puskely, J.H.J. Janssen, , M. Geurts, , A. Roederer, and A. Yarovoy, "Thermal-aware snthesis of 5G base station antenna arrays: an overview and a sparsity-based approach," IEEE Access, vol. 6, pp.5886858882,2018

[7] M. SalarRahimi, E.V.P. Anjos, T. Buss, D.M.M.-P. Schreurs, M. Geurts, G.A. E. Vandenbosch, "A low-cost analog beamforming antenna for $5 \mathrm{G}$ mm-Wave handset applications," In 13th European Conference on Antennas and Propagation (EuCAP 2019), Krakow, Poland, Mar. 2019. 